\title{
NMR Studies of Interactions between C-Terminal Tail of Kir2.1 Channel and PDZ1,2 Domains of PSD95 ${ }^{\dagger}$
}

\author{
Scott Pegan,, Joshua Tan, ${ }^{\S}$ Alan Huang,,$‡$ Paul A. Slesinger, ${ }^{*}, \S$ Roland Riek, $¥$ and Senyon Choe, ${ }^{*}$ \\ Structural Biology and Peptide Biology Laboratories, The Salk Institute, La Jolla, California 92037
}

Received October 26, 2006; Revised Manuscript Received March 2, 2007

\begin{abstract}
Control of surface expression of inwardly rectifying potassium (Kir) channels is important for regulating membrane excitability. Kir2 channels have been shown to interact directly with PDZ-containing proteins in the postsynaptic density (PSD). These scaffold proteins, such as PSD95, bind to Kir2.1 channels via a PDZ-binding motif (T/S-X- $\Phi)$ in the C-terminal tail $\left(\mathrm{SEI}^{428}\right)$. By utilizing a multidimensional solution NMR approach, we show that the previously unresolved structure of Kir2.1 tail (residues 372-428) is highly flexible. Using in vitro binding assays, we determined that shortening the flexible tail of Kir2.1 preceding the C-terminal region (residues 414-428) does not significantly disrupt PDZ binding. We also investigated which amino acids in the Kir2.1 tail associated with PSD95 PDZ1,2 by NMR spectroscopy, revealing that a stretch of $12 \mathrm{C}$-terminal amino acids is involved in interaction with both PDZ domains (residues 417-428). Deletion of the 11 amino acids preceding the C-terminal tail, $\Delta 414-424$, completely disrupts binding to PSD95 PDZ1,2. Therefore, the molecular interfaces formed between PDZ domains and Kir2.1 tail involve regions outside the previously identified binding motif ( $\mathrm{SEI}^{428}$ ) and may be important for additional channel-specific interactions with associating PDZ-containing proteins.
\end{abstract}

Inwardly rectifying potassium $\left(\mathrm{Kir}^{1}\right)$ channels help maintain the resting potential of excitable cells. The property of rectification enables Kir channels to conduct large amounts of potassium ions into the cell, while only permitting small amounts of $\mathrm{K}$ ions to flow out. This small efflux of $\mathrm{K}$ ions helps drive the cell's resting potential near to the $E_{\mathrm{K}}$ of potassium, thereby reducing membrane excitability (1). Several types of Kir channels exist that are gated by cytoplasmic factors, such as G-protein-gated (Kir3), pH-gated (Kir1), and ATP-gated (Kir6) channels. Kir2 channels are unique in that they are constitutively open, requiring only membrane phospholipids to remain open (1). Kir2 channels are important for cardiac and neuronal function. Mutant Kir2.1 channels have been implicated in Andersen's syndrome, a disease characterized by developmental physical disfigurements, periodic paralysis, and cardiac arrhythmias (2). In the brain, mouse knockout studies have shown that

$\doteqdot$ This work was supported by grants from the National Institutes of Health (S.C., P.A.S., R.R.), National Institutes of Health Molecular Biophysics Training Grant (S.P.; GM08326), and the McKnight Endowment for Neuroscience (P.A.S).

* For correspondence: choe@salk.edu, phone (858)453-4100, fax (858) 452-3683; slesinger@salk.edu, phone (858) 453-4100, fax (858)$552-1546$

Structural Biology.

$\S$ Peptide Biology Laboratories.

${ }^{1}$ Abbreviations: PSD, postsynaptic density; PSD95 PDZ1,2, PSD95 fragment (62-249) containing the PDZ1 and PDZ2 domains; PSD95 PDZ1, residues 62-156; PSD95 PDZ2, residues 157-249; SAP, synapse-associated protein; Kir, inwardly rectifying potassium; $\mathrm{PIP}_{2}$, phosphatidylinositol 4,5-bisphosphate; AS, Andersen's syndrome; Kv, voltage-gated potassium (K); Kir2.1 1 , Kir2.1 cytoplasmic domains (4464; 189-428); Kir2.1 , Kir2.1 cytoplasmic domains (44-64; 189-371); IPTG, isopropyl- $\beta$-D-thiogalactopyranoside; DTT, dithiothreitol; wt, wild-type; H8, octahistidine; NMR, nuclear magnetic resonance; CNS, Crystallography \& NMR System Suite. the Kir2.1 channel is key in producing vasodilatation in cerebral blood vessels (3).

Because Kir2 channels are open nearly all the time, cells possess elaborate mechanisms for regulating the number and localization of Kir2 channels in the cell plasma membrane (4). Kir2 channels are expressed in neurons, where they can associate with PSD proteins such as PSD95 (5) (Figure 1a). Within the large family of scaffold proteins, there are four different members of PSD or synapse-associated proteins (SAP): SAP97, SAP102, PSD93 (chapsyn-110), and PSD95 (SAP90) (6). These PSD proteins contain one or more PDZ domains that interact with the C-terminal domains of Kir2 channels and are anchored to the membrane through palmitoylation of two cysteines located at the $\mathrm{N}$-terminus $(4,5$, 7). The PDZ domain is composed of 6 antiparallel $\beta$ strands and two $\alpha$ helices. Typically, a hydrophobic pocket is formed by the $\beta \mathrm{A}-\beta \mathrm{B}$ loop (GLGF) of the PDZ domain that interacts directly with the side chain of a hydrophobic amino acid at the $0^{\prime}$ position (e.g., Val or Ile) of the channel's C-terminal tail, while the Val's free carboxylate binds to the amide hydrogen of a glycine located on $\beta \mathrm{A}(8-10)$. In addition to the Val, association of the PDZ domain also involves the amino acids in the $-1^{\prime}$ and $-2^{\prime}$ positions. The three C-terminal residue motif of the Kir channels for class I PDZ binding is T/S-X- $\Phi$, where $\Phi$ denotes a hydrophobic amino acid (Figure 1b) (9).

Although each of these PSD proteins has structurally similar PDZ domains, the molecular mechanism for the selective association between the channels and PDZ domains is not well understood. For example, Kir2.1, Kir2.2, and Kir2.3 contain similar PDZ-binding motifs, but Kir2.1 and Kir2.3 bind preferentially to PSD95 while Kir2.2 binds to SAP97 $(4,5,11)$. To date, previous studies using indirect 
a)

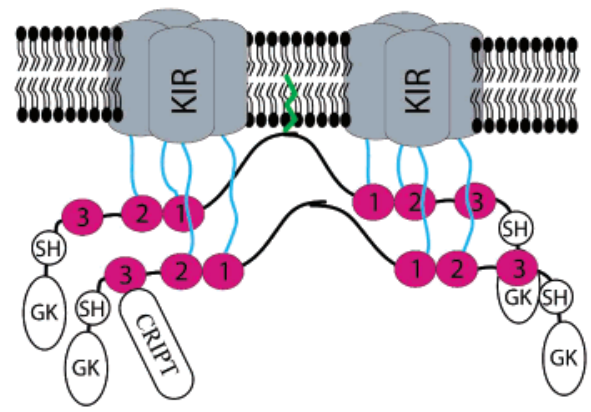

b)

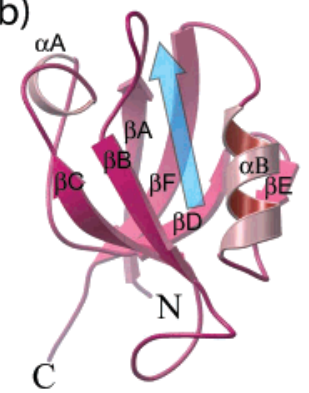

c)

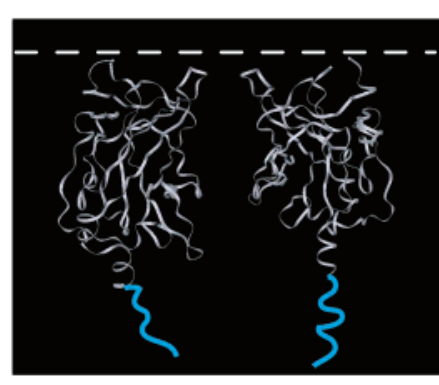

FIGURE 1: PSD95 PDZ2 structural scaffolds and Kir2.1 cytoplasmic domain. (a) Cartoon model of Kir2.1's interaction with PDZ1 and PDZ2 domains of PSD95. Cytoplasmic domain of Kir channels is not depicted for clarity. Palmitoylation is represented in green and flexible Kir2.1 tail region in blue. (b) Structure of PSD95 PDZ2 with translucent blue arrow representing the expected primary binding site for PDZ. (c) Kir2.1 $1_{\mathrm{L}}$ cytoplasmic domain structure in gray shown only with two monomers $\mathrm{A}$ and $\mathrm{B}$. The other two subunits (front and back sides) are removed for clarity. Flexible structural tail region of Kir $2.1_{\mathrm{L}}$ is depicted in blue. The membrane is illustrated as a dotted white line.

mutation and truncation methods have proposed that more than the last three amino acids of the Kir2 C-terminal tail are involved in determining binding to the PDZ motif (5, 11, 12). A structure of this region of Kir2 channels with its cognate PDZ domain could help reveal a more detailed mechanism of binding specificity. Recent structural studies utilizing a $\mathrm{N}-\mathrm{C}$ terminal fusion that contained the entire C-terminal end of Kir2.1, Kir2.1 $1_{\mathrm{L}}$ (44-64 fused to 189428 ), was only visualized up to residue 371 , suggesting that the remaining $57 \mathrm{C}$-terminal tail amino acids were disordered (Figure 1c) (13). In order to determine the exact region of the Kir2 C-terminal segment involved in binding PDZ domains, we have characterized the interactions of Kir2.1 with the PDZ1 and PDZ2 domains of PSD95 by nuclear magnetic resonance (NMR) spectroscopy. We demonstrate that Kir2.1 residues interact with PSD95's PDZ1 and PDZ2 domains more extensively than the last three C-terminal residues, leading to a model for molecular interfaces formed between PDZ domains of PSD95 and Kir2.1, and address the dynamic nature of the unresolved C-terminal tail of Kir2.1.

\section{EXPERIMENTAL PROCEDURES}

Molecular Biology. PSD95 PDZ constructs (PSD95 PDZ1,2 ranging for residues 62-249, and PSD95 PDZ2 for residues 157-249) were cloned from rat PSD95 (100\% homologous to human in this region) into $p H i s 8$ vector, an octahistidinetagged pET21 expression vector (Invitrogen). For Kir2.1 fusion protein constructs (Kir2.1 1 and Kir2.1 $1_{\mathrm{L}}$ ), the cytoplasmic N-terminal and C-terminal domains of mouse Kir2.1 were linked directly in frame by PCR to clone into the $\mathrm{pHis} 8$ vector $(13,14)$. BL21 (DE3) cells were used to express the proteins. GST was fused directly to the C-terminal tail of Kir2.1 (354-428) of Kir2.1 by subcloning into pGEX2T, creating a fusion protein with an intact C-terminal PDZ binding motif (Kir2.1ct). Within this construct, four internal deletion mutants, i.e., $\Delta 384-413$ (deletion of 384-413), $\Delta 394-413$ (deletion of 394-413), $\Delta 404-413$ (deletion of 404-413), and $\Delta 414-424$ (deletion of 414-424), and one additional $\mathrm{C}$-terminal deletion mutant, $\Delta C$ (deletion of $\mathrm{SEI}^{428}$ ), were generated by PCR.

Expression of Proteins and Generation of Peptide. ${ }^{15} \mathrm{~N}-$ and ${ }^{15} \mathrm{~N},{ }^{13} \mathrm{C}$-labeled samples were obtained by expressing in bacteria grown at $37{ }^{\circ} \mathrm{C}$ to $0.6 \mathrm{OD}$ in LB media. Cells were spun down, washed with PBS, spun again, and resuspended in minimal media containing appropriate isotope labels (15). Half an hour after growing at $37{ }^{\circ} \mathrm{C}$ post media exchange into minimal media, the cells were induced by $0.5 \mathrm{mM}$ IPTG and grown for $3 \mathrm{~h}$ at $30{ }^{\circ} \mathrm{C}$. Nonlabeled proteins for NMR were expressed in cells grown in TB at $37{ }^{\circ} \mathrm{C}$ to $0.6 \mathrm{OD}$ optical density $\left(A_{600}\right)$ and induced with $0.5 \mathrm{mM}$ IPTG for 3 h. For protein purification, cells were lysed in $0.5 \mathrm{M} \mathrm{NaCl}$, $5 \mathrm{mM}$ Tris- $\mathrm{HCl}, \mathrm{pH} 8.5,10 \%$ glycerol, $7 \mathrm{mM} \beta$-mercaptoethanol (lysis buffer), and $1 \mathrm{mg}$ of lysozyme per $100 \mathrm{~mL}$ of lysate. The supernatant from the $96,000 \mathrm{~g}$ centrifugation of the cell lysate was loaded on a nickel-affinity column (Qiagen) and eluted with $200 \mathrm{mM}$ imidazole. Thrombincleaved protein samples were separated by S200 and S75 Sepharose chromatography, and concentrated to $10 \mathrm{mg} \mathrm{mL}^{-1}$ for Kir2.1 $1_{\mathrm{L}}$. For GST fused proteins, bacteria were grown to $0.6 \mathrm{OD}$, induced with $400 \mu \mathrm{M}$ IPTG at $28{ }^{\circ} \mathrm{C}$ for $3 \mathrm{~h}$, and purified using standard techniques for GST-tagged and octahistidine-tagged proteins (16). Kir2.1 $1_{\mathrm{L}}$ tail peptide was generated using solid-phase synthesis, Fmoc cleavage/ deprotection, and purification using a C8 reverse phase, and confirmed with MALDI TOF mass-spectroscopy.

NMR Experiments. The NMR experiments were carried out on a Bruker Avance 700 spectrometer at $25^{\circ} \mathrm{C}$ by using protein in solutions of $85 \% \quad \mathrm{H}_{2} \mathrm{O} / 15 \% \mathrm{D}_{2} \mathrm{O}$ containing $150 \mathrm{mM} \mathrm{NaCl}, 2 \mathrm{mM}$ DTT, $20 \mathrm{mM} \mathrm{KPO} / \mathrm{NaPO}_{4}$ at $\mathrm{pH}$ 6.5. Protein stock concentrations were determined by absorbance at $280 \mathrm{~nm}$ performed in triplicate at three dilutions. Final sample concentrations were determined by a calculated dilution of protein stock and are represented in monomeric molar values. Synthetic peptide concentration was by dry weight. The $\left[{ }^{15} \mathrm{~N},{ }^{1} \mathrm{H}\right]$-TROSY spectrum $(17)$ of ${ }^{15} \mathrm{~N}$-labeled Kir2. $1_{\mathrm{L}}$ was measured at a protein concentration of $0.25 \mathrm{mM}$, with $t_{1, \max }=88 \mathrm{~ms}, t_{2, \max }=98 \mathrm{~ms}$, a data size of $512 \times$ 1024 complex points, and an overall recording time of $3 \mathrm{~h}$ at $30{ }^{\circ} \mathrm{C}$. TROSY based steady-state ${ }^{15} \mathrm{~N}\left\{{ }^{1} \mathrm{H}\right\}$-NOEs of the backbone amide groups were measured with $0.25 \mathrm{mM}$ solution of Kir2 $21_{\mathrm{L}} \cdot\left[{ }^{15} \mathrm{~N},{ }^{1} \mathrm{H}\right]$-TROSY experiments to measure Kir2.1 1 / PDZ binding were carried out using solutions containing $0.25 \mathrm{mM}{ }^{15} \mathrm{~N}$-labeled $\mathrm{Kir} 2.1_{\mathrm{L}}$ and unlabeled PSD95 PDZ domains 1 and 2 (62-249; PSD95 PDZ1,2) or PSD95 PDZ2 (157-249; PDZ2) concentrations of 3.9, 7.83, $11.7,15.6,19.5,27.0,31.0,35.2,62.5,125.0$, and $250.0 \mu \mathrm{M}$ for $3 \mathrm{~h}$ at $30{ }^{\circ} \mathrm{C}$. For binding experiments with PSD95 PDZ2 and Kir2.1 $1_{\mathrm{L}}$ tail peptide, solutions containing $0.682 \mathrm{mM}$ of ${ }^{15} \mathrm{~N}$-labeled PSD95 PDZ2 (157-249) and unlabeled Kir2.1 tail peptide (414-428) at concentrations of $0.0107,0.0213$, $0.0426,0.0853,0.171,0.341$, and $0.682 \mathrm{mM}$ were measured over $3 \mathrm{~h}$ at $30^{\circ} \mathrm{C}$. The NMR data sets were processed with 

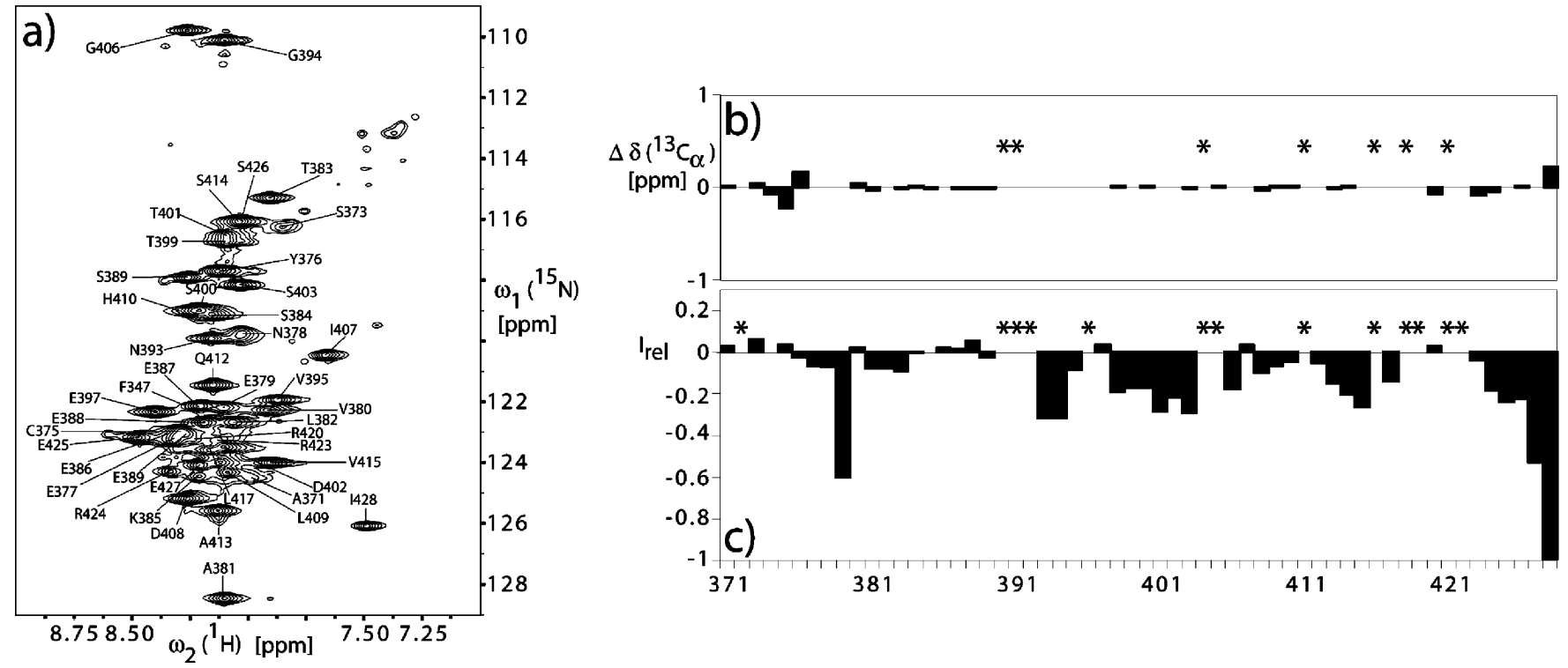

FIGURE 2: Assignment and dynamics of Kir2 $.1_{\mathrm{L}}$ cytoplasmic C-terminal tail region $371-428$. (a) $\left[{ }^{15} \mathrm{~N},{ }^{1} \mathrm{H}\right]-\mathrm{TROSY}$ spectrum with assignments of residues $371-428$ in Kir2 $11_{\mathrm{L}}$. (b) Chart of $\left[{ }^{15} \mathrm{~N},{ }^{13} \mathrm{C}\right]$ HNCA deviations of ${ }^{13} \mathrm{C}_{\alpha}$ shift from random coil values. (c) Chart of steady state ${ }^{15} \mathrm{~N},{ }^{1} \mathrm{H}$-NOE experiment results. $I_{\text {rel }}$ is derived from dividing the cross-peak intensity of the ${ }^{15} \mathrm{~N}\left\{{ }^{1} \mathrm{H}\right\}$-NOE experiment by the corresponding intensities of the reference experiment.

the program PROSA (18) and analyzed with the program CARA (19).

The sequential assignment of the C-terminal tail of Kir2.1 $1_{\mathrm{L}}$ was obtained using the triple resonance experiment $\mathrm{HNCA}$ codedCO, a ${ }^{15} \mathrm{~N}$-resolved $\left[{ }^{1} \mathrm{H},{ }^{1} \mathrm{H}\right]$-NOESY experiment with $200 \mathrm{~ms}$ mixing time, and a ${ }^{15} \mathrm{~N}$-resolved $\left[{ }^{1} \mathrm{H},{ }^{1} \mathrm{H}\right]$-TOCSY following the standard procedure for ${ }^{13} \mathrm{C},{ }^{15} \mathrm{~N}$-labeled proteins (20-23).

In Vitro Binding Assay. Far Western in vitro binding assays were used to assess binding of Kir2.1 C-terminal tails to PSD95 PDZ1,2 (24). Briefly, GST-fused proteins ( $1 \mu \mathrm{g})$ were separated by SDS-PAGE, transferred to nitrocellulose, and then stained with Ponceau-S (Sigma) to visualize transferred proteins. Blots were placed in blocking buffer containing $2.5 \%$ BSA in TBST (25 mM Tris pH 7.4, $150 \mathrm{mM} \mathrm{NaCl}, 2$ $\mathrm{mM} \mathrm{KCl}, 0.05 \%$ Tween-20) and incubated overnight at 4 ${ }^{\circ} \mathrm{C}$. Fusion protein probe (100 or $250 \mathrm{nM}$ for H8-PSD95 PDZ1,2) was added to blocking buffer and incubated for 1 $\mathrm{h}$ on a shaker at room temperature. To detect bound protein, Western immunobloting was carried out with anti-HisProbe HRP conjugated (Pierce; 1:2500 dilution) antibodies in TBST- $0.05 \%$ Tween-20. Blots were washed, incubated with SuperSignal ECL reagents (PIERCE), and exposed to BioMax XAR film (Kodak) for a range of times $(0.1-5 \mathrm{~min}$ ). Blots were quantified by measuring the OD of anti-H8 band and normalizing by the OD of the Ponceau-S band, which adjusted differences in transfer and estimation of total protein. Three experiments were pooled by determining the change in OD for mutants relative to that for GST-Kir2.1-ct and averaging together (mean \pm SEM).

PSD95 PDZ2 Homology Model. A homology model of PSD95 PDZ2 with a seven-residue Kir2.1 C-terminal peptide was generated based on experimentally guided placement of the peptide as well as global model energy minimization. Using Modeller v8, we used the X-ray structure of rat PDZ95 PDZ3 domain/cript peptide complex as a template to model PSD95 PDZ2 and the corresponding residues Glu 425, Ser 426, Glu 427, and Ile 428 of Kir2.1. The lowest energy model was selected, and Kir2.1 residues Leu 422, Arg 423, and Arg 424 were added to the model based on possible CA orientations and $\left[{ }^{15} \mathrm{~N},{ }^{1} \mathrm{H}\right]$-TROSY data. The model of PSD95 PDZ2 in complex with 7 residues of Kir2.1 (Leu422-Ile428) was then energy minimized using a conjugate gradient minimization program of Crystallography \& NMR System Suite (CNS) with default settings.

\section{RESULTS}

Assignment of the Kir2.1 $1_{L}$ Cytoplasmic C-Terminal Tail 371-428. To assign the presumed C-terminal tail (371428 ) of the tetrameric $120 \mathrm{kDa}$ cytoplasmic domain of Kir2. $1_{\mathrm{L}}$, we made use of different relaxation properties of the structural domain versus the presumed tail. Normally, solution NMR analysis on the entire $120 \mathrm{kDa}$ homotetrameric complex is difficult, since large proteins tumble slowly in solution, causing enhanced relaxation and concomitantly resulting in reduced signal. Hence, the signal from the presumed flexible tail's $\mathrm{N}-\mathrm{H}$ moiety should be considerably stronger than the rest of the protein and thus be readily measurable by solution NMR. To exploit this possibility, we made ${ }^{15} \mathrm{~N}$-labeled Kir2. $1_{\mathrm{L}}$ and then performed a $\left[{ }^{15} \mathrm{~N},{ }^{1} \mathrm{H}\right]$ TROSY experiment at $298 \mathrm{~K}$ to visualize the $\mathrm{N}-\mathrm{H}$ moieties of the protein (17). The $\left[{ }^{15} \mathrm{~N},{ }^{1} \mathrm{H}\right]$-TROSY showed 44 crosspeaks including two glycine residues (Figure 2a). This matched exactly with the number of glycines present in the tail of Kir2.1 $1_{\mathrm{L}}$. Furthermore, we performed the same experiment on the Kir2.1 (residues 1-64 fused to 189-371) which lacks the C-terminal tail residues of interest. Indeed, the observed cross-peaks in the TROSY spectrum of ${ }^{15} \mathrm{~N}$-labeled Kir2. $1_{\mathrm{L}}$ are absent in the spectrum of the truncated construct (data not shown).

With the $\left[{ }^{15} \mathrm{~N},{ }^{1} \mathrm{H}\right]$-TROSY results confirming our hypothesis, we moved on to assigning the peaks to their 51 corresponding residues, excluding six prolines present in the Kir2. $1_{\mathrm{L}}$ tail. Through the use of triple resonance and ${ }^{15} \mathrm{~N}$ resolved $\left[{ }^{1} \mathrm{H},{ }^{1} \mathrm{H}\right]-\mathrm{NOESY}$ experiments coupled with ${ }^{15} \mathrm{~N}$ resolved TOCSY experiments to confirm connectivity and 
a)

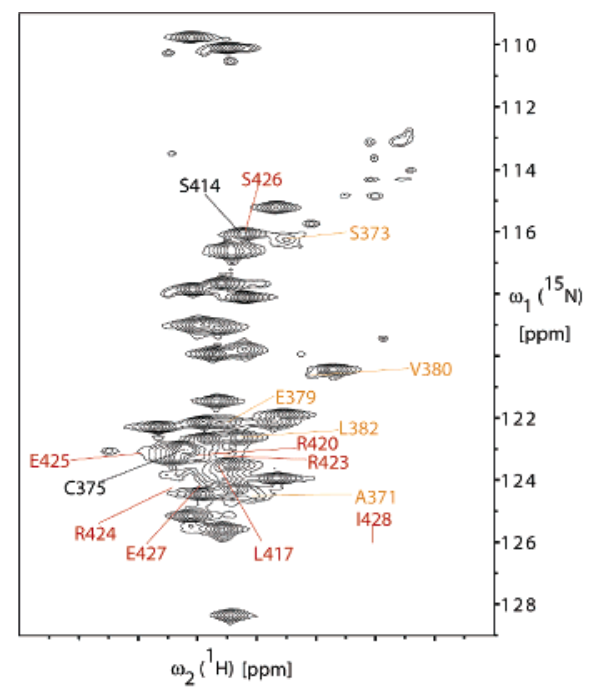

c)

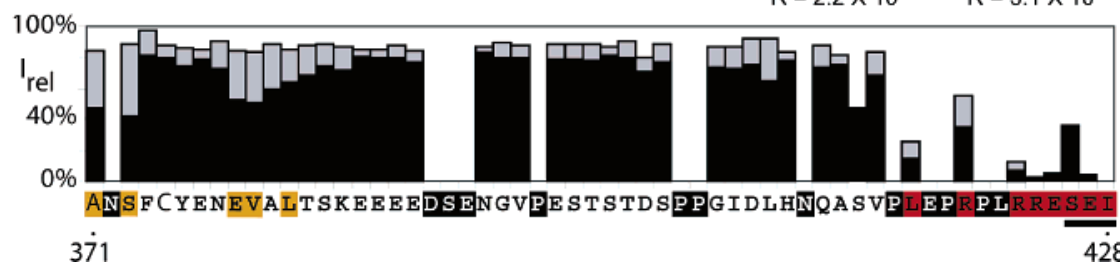

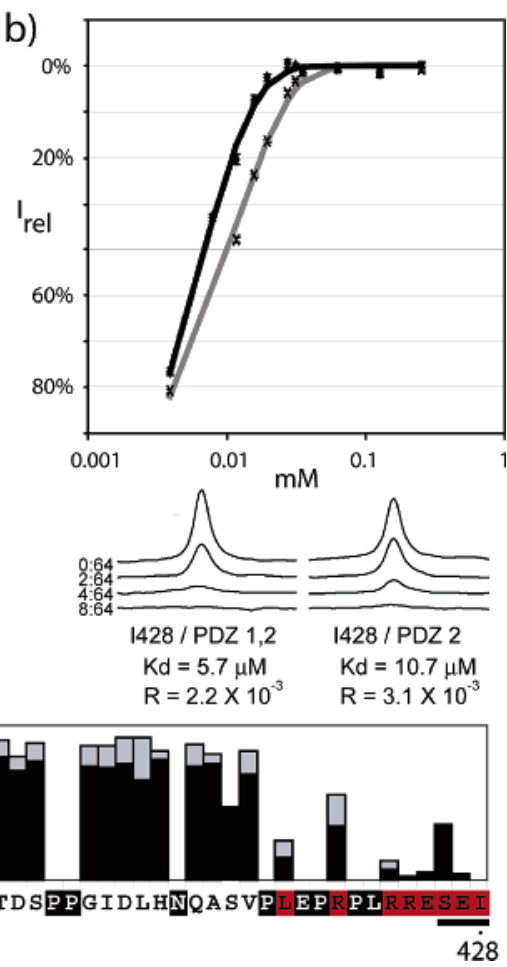

b)

FIGURE 3: Binding footprint and binding measurements of PSD95 PDZ1,2 and PSD95 PDZ2 alone. (a) $\left[{ }^{15} \mathrm{~N},{ }^{1} \mathrm{H}\right]-\mathrm{TROSY}$ of Kir2. $1_{\mathrm{L}}$ when combined with PSD95 PDZ1,2 at 1:1 molar ratio. Residues with signal lost to less than $45 \%$ level are labeled in red; between $45 \%$ and $60 \%$ in orange. (b) Graph illustrates the fits for integrals of peak I428 amide versus concentration of the PSD95 PDZ1,2 (black) and PSD95 PDZ2 (gray) constructs at molar ratios 1:64, 2:64, 3:64, 4:64, 5:64. 8:64, 16:64, 32:64, and 1:1 (PSD95 PDZ1,2) and 1:64, 3:64, 4:64, 5:64, 8:64, 16:64, 32:64, and 1:1 (PSD95 PDZ2). Error bars represent standard deviation of noise from each $\left[{ }^{15} \mathrm{~N}\right.$, $\left.{ }^{1} \mathrm{H}\right]-\mathrm{TROSY}$ spectral data point. The $x$-axis is in log scale with the 0:64 data point removed for clarity. Shown below the plots are 1D traces of the I428 amide signal for a subset of data: 0:64, 2:64, 4:64, and 8:64. (c) Percent of change of peak intensities in $\left[{ }^{15} \mathrm{~N},{ }^{1} \mathrm{H}\right]$-TROSY spectra per residue between the PDZ1,2-bound/PDZ1,2-unbound Kir2.1 $\mathrm{L}_{\mathrm{L}}$ (black) and PDZ2-bound/PDZ2-unbound Kir2.1 (gray) at 1:1 molar ratios. $I_{\text {rel }}$ is calculated by [(unbound signal intensity) - (bound signal intensity)]/(unbound signal intensity). For those five amino acids (424-428) where black bars obscure gray bars, the level of quenching of PSD95 PDZ2 residues are within 1\% of those PSD95 PDZ1,2 values with the exception of S426. S426's quenching level was $\sim 5 \%$ less that of PSD95 PDZ1,2. Amino acids over the sequence segment $371-428$ of Kir2.1 $1_{\mathrm{L}}$ with strong binding region (large signal quenching) are highlighted in red, nonassigned residues boxed in black. Orange residues represent upstream residues that are moderately affected by binding.

side chain identity, we assigned 44 distinct peaks of the 51 possible residues in the tail, resulting in $86 \%$ coverage of the possible $\mathrm{N}-\mathrm{H}$ moieties. (Figure $2 \mathrm{a})(20,22)$.

C-Terminal Tail of Kir2.1 Is Highly Flexible with No Secondary Structure. ${ }^{13} \mathrm{C}_{\alpha}$ chemical shift analysis shows on average low divergence from the values of a random coil indicating a lack of secondary structure for this region (Figure 2b). Only, the C-terminal residue (I428) had a positive shift, which is attributed to the influence of the additional carboxyl group of the $\mathrm{C}$-terminus. Two other residues, Phe 375 and Tyr 376, showed small shift deviation, but again no substantial and sustained positive ( $\alpha$ helical) or negative ( $\beta$ sheet) deviations could be observed from neighboring residues (Figure $2 b$ ).

In order to confirm the flexibility and lack of secondary structure; we conducted a steady state ${ }^{15} \mathrm{~N}\left\{{ }^{1} \mathrm{H}\right\}$-NOEs experiment to measure the effective rotational correlation transfer of individual $\mathrm{N}-\mathrm{H}$ vectors. Positive values, which correspond to a $\tau_{\mathrm{c}}>1 \mathrm{~ns}$, would have indicated structured regions, whereas negative values correspond to a $\tau_{\mathrm{c}}<1 \mathrm{~ns}$ and are indicative of high flexibility (32). Figure $2 \mathrm{c}$ summarizes that the residues were either negligibly positive or substantially negative, confirming that the tail region of the Kir2.1 channel is inherently flexible and does not possess any secondary structure.
PSD95 PDZ-Binding Region of Kir2.1 $1_{L}$ Tail. Given the structural dynamics of the tail, we shifted our attention to the binding of PDZ domains to the Kir $2.1_{\mathrm{L}}$ construct. We employed the use of two PSD95 constructs: one containing both PDZ1 and PDZ2 domains (62-249; PSD95 PDZ1,2) and the other being just PSD95 PDZ2 (157-249; PSD95 PDZ2). Interaction of Kir2.1 $1_{\mathrm{L}}$ tail residues with either PDZ construct would have their free-state $\left[{ }^{15} \mathrm{~N},{ }^{1} \mathrm{H}\right]$-TROSY signal quenched due to the reduction of their flexibility and possibly shifted due to a change of the electronic environment. In conjunction, the bound state of tail residues involved with binding the PDZ may not be visible as a result of the larger complex size of the Kir2.1 $1 \mathrm{~L} / \mathrm{PDZ}$ complex, $156 \mathrm{kDa}$, or if exchange between the bound and free states is slow or immediate (25).

Upon addition of the PSD95 PDZ1,2 to ${ }^{15} \mathrm{~N}$-labeled Kir2. $1_{\mathrm{L}}$ in molar ratios ranging from 1:64 to $1: 1$, we find that more than the last three amino acid PDZ motif show binding based on the quenched signals. More specifically, residues Leu 419, Arg 423, Arg 424, Glu 425, Glu 427, and Ile 428 show significant binding as their signal was quenched between $88 \%$ and $100 \%$ (Figure 3). Interspersed among these highly quenched residues are Arg 420 and Ser 426, which exhibit quenching greater than $60 \%$. The apparent reduction of quenching in these two residues is due to their $\left[{ }^{15} \mathrm{~N},{ }^{1} \mathrm{H}\right]-$ 
TROSY peaks overlapping with other tail residues. Upon close examination of the positions of the $\left[{ }^{15} \mathrm{~N},{ }^{1} \mathrm{H}\right]$-TROSY peak maximum for Arg 420's and Ser 426's N-H moieties, we were able to discern that their overlap with nonquenching $\mathrm{N}-\mathrm{H}$ moieties of Cys 375 and Ser 414 respectively elevates their experimental baselines. Apart from Ser 414 and its neighbor, V415, all eight assigned out of ten potentially observable residues (Glu 418 and Leu 422 could not be assigned) across the last 12 C-terminal amino acids, 417428, of Kir $2.1_{\mathrm{L}}$ suggests that it is this 12 residue segment that interacts with PSD95 PDZ1,2.

By plotting the degree of quenching observed for the 12 amino acid region at varying substoichiometric concentrations of PSD95 PDZ1,2 and PSD95 PDZ2, we observe that the PDZ domain's interaction with the tails of the Kir2.1 follows a typical binding curve. Consequentially, we used I428's quenching as the basis to estimate apparent $K_{\mathrm{d}}$ with respect to PSD95 PDZ1,2 and PSD95 PDZ2 (Figure 3b). The identification of a $K_{\mathrm{d}}$ in slowly exchanging macromolecular complexes by measuring the quenching of NMR signals must be made with caution, since line broadening might appear due to the possible chemical shift and relaxation differences between bound and unbound state (25). However, under the assumption that during the polarization transfers of the $\left[{ }^{15} \mathrm{~N},{ }^{1} \mathrm{H}\right]$-TROSY experiment no or only little signal is lost due to these additional variable relaxation mechanisms, a reasonable $K_{\mathrm{d}}$ can be estimated by the decay of the volume of the cross-peaks versus varying substoichiometric concentrations. This assumption is justified in the system studied, since a ${ }^{1} \mathrm{H}$ line broadening analysis suggests signal loss during the polarization transfer of less than $15 \%$ (data not shown). Based on this reasoning, our observations indicate that the (apparent) $K_{\mathrm{d}}$ for the PSD95 PDZ1,2 is $5.7 \mu \mathrm{M}$ and is approximately half that of PSD95 PDZ2, indicating that its tighter interaction may arise from the additional PDZ1 domain. These estimates of $K_{\mathrm{d}}$ are approximations of binding affinity because the significant fraction of $0.25 \mathrm{mM} \mathrm{Kir} 2.1_{\mathrm{L}}$ used in the experiment does not contribute to the signal and acts as the binding counterpart of PDZ. The reason can be biochemical, for instance, the tail being unavailable due to its buried location or the protein being mildly aggregated. Nevertheless, the $\sim 50 \%$ signal being quenched by the PDZ domains at the estimated $K_{\mathrm{d}}$ values indicates that these $K_{\mathrm{d}}$ estimates are reasonable approximations. More importantly, the $K_{\mathrm{d}}$ difference between PDZ1,2 and PDZ2 is less dependent on this unknown factor and thus reliable. This can be seen when plotted on a log graph as a clear shift to the right such that PSD95 PDZ1,2 binds approximately 2-fold more tightly than PSD95 PDZ2 (Figure 3b). Figure 3c summarizes percent change of peak intensities in $\left[{ }^{15} \mathrm{~N},{ }^{1} \mathrm{H}\right]-$ TROSY spectra per residue between the PSD95 PDZ1,2bound and PSD95 PDZ1,2-unbound Kir2.1 L.

In Vitro Binding Assay. We used an in vitro overlay binding assay to characterize further the nature of the flexible tail and the effect of shortening the tail between the $\alpha B$ helix (C-terminus of the tetrameric assembly core) and the C-terminal PDZ-binding motif (SEI ${ }^{428}$ ) of Kir2.1 (Figure 4a). As expected, the C-terminal tail of Kir2.1 (P354-I428) by itself exhibits strong binding to PSD95 PDZ1,2 by this method, in contrast to GST alone (Figure 4b). Deleting the last three amino acids of the PDZ binding motif $(\Delta C)$ eliminated the binding to $100 \mathrm{nM}$ PSD95 PDZ1,2. We then studied the effect of internal deletions ranging from 10 to 30 amino acids, in GST-Kir2.1ct. Kir2.1 deletion mutants $\Delta 384-413, \Delta 394-413$, and $\Delta 404-413$ all exhibited similar or stronger binding than intact C-terminal tail (Figure $4 b$ ). In contrast, a deletion of 11 amino acids just before the ESEI sequence, $\Delta 414-424$, reduced the binding to background levels (Figure 4b,c). A higher concentration of probe $(250 \mathrm{nM})$ and longer exposure to film $(5 \mathrm{~min})$ did not reveal a detectable amount of H8-PSD95 PDZ1,2 protein bound to either GST-Kir2.1 $\Delta \mathrm{C}$ or GST-Kir2.1 $\Delta 414-424$ (not shown). We cannot rule out that these deletion mutants retain some very low affinity PDZ binding, but it is nevertheless considerably less than wild-type or other deletion mutants. Interestingly, the construct with the shortest tail appeared to bind better than wild-type. These findings support the conclusion that the region of 417-428 identified by NMR analysis is also essential for PDZ binding of Kir2.1. Furthermore, data show that the length of the peptide chain between the $\alpha \mathrm{B}$ helix and the 12-residue PDZ binding motif does not appear to have a significant role in determining PDZ binding.

Kir2.1 $1_{L} 414-428$ Peptide Binding to PSD95 PDZ2. Based on the NMR experiments and in vitro binding assay performed on the cytoplasmic domains of Kir2.1, we used a C-terminal 15 residue synthetic peptide composed of Kir2.1 residues $414-428$ to probe against one of the single PDZ domains to gain a greater understanding of specific amino acid interactions between the expanded PDZ motif and a PDZ domain. As PSD95 PDZ2's assignment was readily available and was already subcloned into a his-tag construct, we chose to utilize PSD95 PDZ2 for this study (26). By labeling PSD95 PDZ2 with ${ }^{15} \mathrm{~N}$ while titrating the unlabeled peptide, we followed peak shifts and intensities to identify residues of PSD95 PDZ2 that were affected by peptide binding (Figure 5). As expected, the bulk of residues that were affected by binding the 15-residue peptide are clustered near the binding site previously identified by the 4-residue cripto peptide. However, Asp 222, Met 224, Glu 226, Tyr 190, and Thr 192 of PSD95 PDZ2 also showed significant shift and signal loss, which were not identified previously. Based on these peak permutations, Kir2.1 $1_{\mathrm{L}}$ residues implicated in binding PDZ domains and the previous structure of PDZ3 bound to a small peptide from the protein cripto, we qualitatively outlined putative PSD95 PDZ2 interaction site with Kir2.1 tail (9) (Figure 5b).

\section{DISCUSSION}

Flexible Kir2.1 C-Terminal Tail. Utilizing two different analyses with NMR, we determined that the C-terminal tail lacks secondary structure, similar to that of a random coil. The finding that a flexible region of a channel is immobilized upon binding is not uncommon. For example, the voltage gated potassium $(\mathrm{Kv})$ channel has a naturally occurring flexible N-terminal domain that produces rapid channel inactivation by inserting into the channel pore (27). Lack of structure in the C-terminal chain of Kir2.1 channels does allow for easy access to an endoplasmic reticulum (ER) export signal located at the beginning of the chain (28). The flexibility of the chain and the number of residues between the ER export tag and the three C-terminal amino acids in the PDZ motif also raise the possibility that this region plays additional important functions. Generally, the backbone 
a)

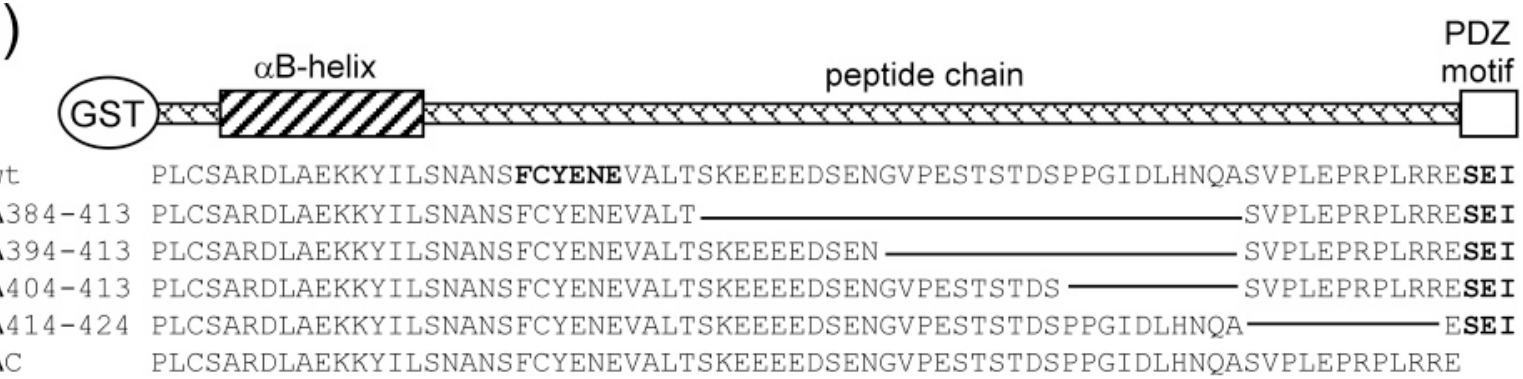

b)

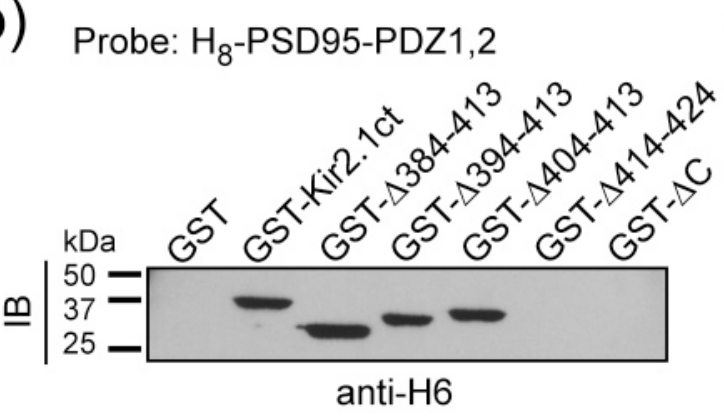

C)

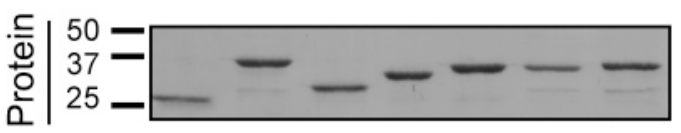

Ponceau

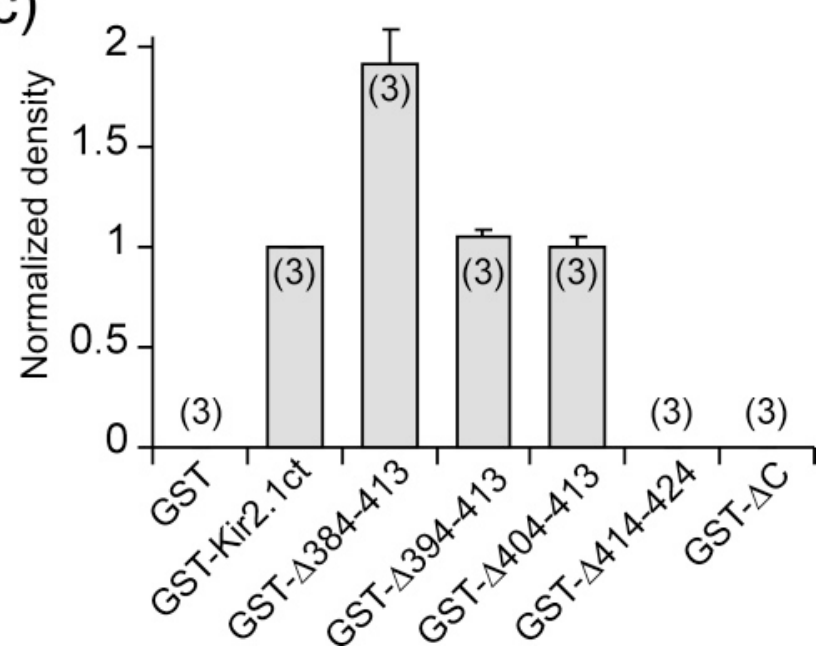

FIGURE 4: Role of linker and C-terminal tail of Kir2.1 in PDZ binding. (a) Schematic rendering of the C-terminal tail for Kir2.1 and the amino acid sequences of different deletion mutants are shown. The length of the peptide chain linker was shortened by making 10,20 , and 30 amino acid deletions. "FYCENE" sequence highlights ER export signal (31). (b) In vitro overlay binding assay. Each of the indicated GST proteins $(1 \mu \mathrm{g})$ was separated by SDS-PAGE, transferred to nitrocellulose, and probed with purified H8-PSD95 PDZ1,2 (100 nM). Bound protein was visualized with anti-H6 antibody (immunoblot -IB). Protein transferred to nitrocellulose visualized by Ponceau S stain (bottom). Shortening the peptide chain did not reduce binding. However, removing the last three $(\Delta \mathrm{C})$ or the 11 amino acids before the ESEI $^{428}$ eliminated binding to $100 \mathrm{nM}$ H8-PSD95 PDZ1,2. (c) Quantification of in vitro binding assay (see Methods for details). Three experiments were pooled by determining the change in OD for mutants relative to that for GST-Kir2.1-ct and averaging together (mean \pm SEM).

length of one $\mathrm{C}_{\alpha}$ to the next is $3.5 \AA$, yielding a Kir2.1 channel tail of $\sim 57$ residues that could elongate up to $\sim 199 \AA$ A. Although an estimate, the length of the tail combined with the $48 \AA$ of the cytoplasmic domains of Kir2.1 is more than sufficient to span the distance observed by cryo EM studies, $120 \AA$, between the membrane and PSD95 scaffold of other PSD95 binding membrane proteins (28). With approximately $127 \AA$ to spare, the additional length of the tail leaves room for flexibility in order for cells to endure mechanical stresses and an extended PDZ recognition site of 12 residues (42 ̊).

PDZ Recognition and Binding Region. Recent studies of other proteins with PDZ binding motifs have illustrated that residues outside the established three amino acid motif can directly interact with PDZ domains up to the -7 ' tail position $(12,29,30)$. However, we observe that the interaction is more extensive in the Kir2.1 $1_{\mathrm{L}}$ tail as it interacts with PDZ domains through a 12 amino acid segment. This is in line with results from a previous yeast two-hybrid truncation experiment, where loss of a 13 residue segment directly before the three residue PDZ binding motif abolished PDZ binding capability from the tail (5). Others have suggested that a second class II PDZ binding motif may exist in the tail of Kir2.3, which is homologous to ENGV in Kir2.1 channels (5). Although the glutamate of this sequence was not assigned during this study, the remaining three residues underwent no quenching in the presence of either the PSD95 PDZ2 or PSD95 PDZ1,2 proteins (Figure 3c). Furthermore, deleting this region of the chain did not eliminate binding to the PDZ domain.

The involvement of residues beyond the last three residues allows for encoding specificity determinants of PDZ domains more extensively. The homology model of PSD95 PDZ2 with the 7 C-terminal peptide of Kir2.1 allows us to speculate on the interaction of the extended region. For instance, Arg423 and Arg424 potentially form favorable bonds with Glu 226 and Asp 222 respectively (Figure 5c). This electrostatic interaction is homologous to that observed in Gee et al. where a negative charge in $\alpha \mathrm{B}$ helix of PDZ domains interacts with a positively charged amino acid at the "-4" position (29). These two potential salt bridges formed by Arg 423 and Arg 424 of Kir2.1 channels could provide additional binding stability and explain preference of Kir2.1 and Kir2.3 channels binding PDZ1 and PDZ2 domains of PSD95 over the PDZ3 domains. In contrast to the PDZ2's structurally positioned negatively charged residues, Asp 222 and Glu 226, PDZ3 has two polar residues, asparagine and glutamine. As for the affinity differences within the Kir2 channel family (Kir2.1-Kir2.4), the last six tail residues are highly homologous (RRES(E/A)I). There- 
a)

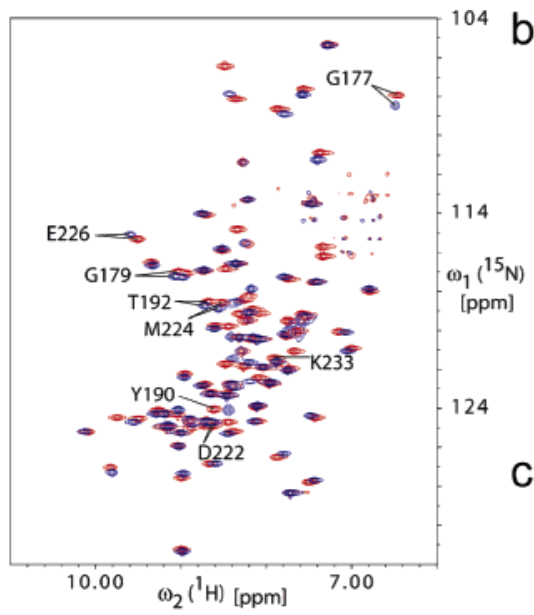

b)

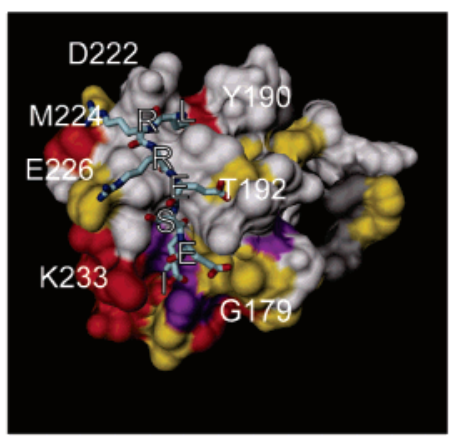

c)

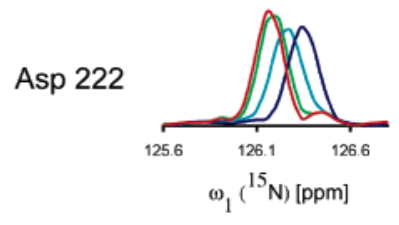

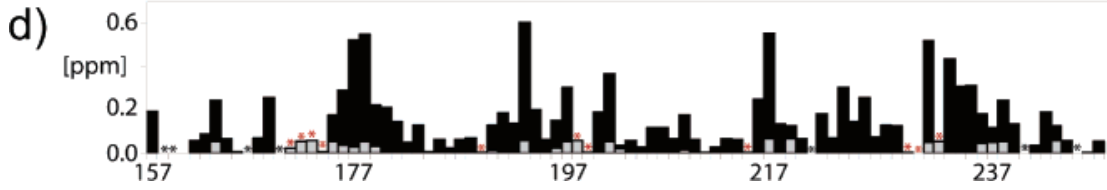

FIGURE 5: PSD95 PDZ2 binding footprint of Kir2.1 (414-428) peptide. (a) $\left[{ }^{15} \mathrm{~N},{ }^{1} \mathrm{H}\right]-\mathrm{TROSY}$ of PSD95 PDZ2 without (blue) and with (red) at a 1:1 molar ratio of Kir2.1 (414-428) peptide. (b) Model of PSD95 PDZ2 binding to Kir2.1's last seven amino acids (VRRESEI). Residues are colored corresponding to their shifts upon binding peptide, $0.45 \mathrm{ppm}$ or greater (purple), $0.3-0.44 \mathrm{ppm}$ (orange), $0.15-0.29$ ppm (yellow), and 0-0.15 ppm (white). Residues not assigned are colored in gray, and residues that were broadened are in red. (c) Peak graphics are slice windows of 0:1 (red), 1:8 (green), 1:2 (teal), and 1:1 (blue) of molar ratios of peptide to PSD95 PDZ2 through the 0:1 $\left[{ }^{15} \mathrm{~N},{ }^{1} \mathrm{H}\right]$-TROSY spectrum along the ${ }^{15} \mathrm{~N}$ axis. (d) Panel illustrates peak shift in ppm of peak locations from unbound to bound PSD95 PDZ2 with peptide added at 1:16 (gray) and 1:1 (black) molar ratios. Red asterisks mark residues that have complete signal loss at 1:1 molar ratio. Black asterisks denote residues not observed during the experiment.

fore, we suggest that the region between residues 417 and 422 plays an additional role by encoding PDZ-binding specificity among related Kir2 channels. This region is extremely diverse in composition. For example, Leu 422 of Kir2.1 is tyrosine in Kir2.2. In the model, Leu 422 appears to fit fairly well into a hydrophobic pocket formed by PSD95 PDZ2's Tyr 190, but not the tyrosine of the Kir2.2 (Figure 5b). Furthermore, both Kir2.3 and Kir2.1 contain a proline at the -13 position, which is a valine in Kir2.2. Since this Pro 416 lacks an NMR signal for lack of its amide proton, its interaction with Kir cannot be measured by our experiment. Nevertheless, its neighboring residue, Leu 417, undergoes $80 \%$ quenching upon binding to PDZ domains and thus it is an excellent candidate to play a role in generating a hydrophobic pocket and possibly differentiating binding between PSD95 and SAP97 motifs. These speculations based on the model await experimental verification.

Enhanced Apparent Affinity between Kir $2.1_{L}$ and PSD 95 $P D Z 1,2$. The difference in binding affinities between the Kir2.1L/PSD95 PDZ2 and tail peptide/PSD95 PDZ2 suggests that the interaction between Kir2.1 and PSD95 in cell membrane is enhanced because of the spatial restriction of the four tails. Whereas the last residue in Kir2.1 1 , Ile 428, has an apparent affinity for PSD95 PDZ2 of $10.7 \mu \mathrm{M}$, PSD95 PDZ2 residues do not show comparable affinity even in the presence of the tail peptide at a concentration of nearly full binding. There are other factors to consider. Even though the $K_{\mathrm{d}}$ of PSD95 PDZ2 to a single tail is weaker than the apparent $K_{\mathrm{d}}$, the local concentration of four tails created by the tetramerization of the channel can contribute to tight interaction. Furthermore, the apparently higher affinity of PSD95 PDZ1,2 $(5.7 \mu \mathrm{M})$ suggests that the tails may interact with both PDZ domains of PSD95 within or across the protein chain so that such interactions of the multiple tails to multiple PDZ domains coupled through the markedly extended PDZ binding region will contribute to forming the tight and selective interactions required for channel clustering.

\section{REFERENCES}

1. Nichols, C. G., and Lopatin, A. N. Inward rectifier potassium channels, Annu. Rev. Physiol. 1997, 59, 171-191.

2. Plaster, N. M., et al. Mutations in Kir2.1 cause the developmental and episodic electrical phenotypes of Andersen's syndrome, Cell 2001, 105 (4), 511-519.

3. Zaritsky, J. J., et al. Targeted disruption of Kir2.1 and Kir2.2 genes reveals the essential role of the inwardly rectifying $\mathrm{K}(+)$ current in $\mathrm{K}(+)$-mediated vasodilation. Circ. Res. 2000, 87 (2), 160166.

4. Cohen, N. A., et al. Binding of the inward rectifier $\mathrm{K}+$ channel Kir 2.3 to PSD-95 is regulated by protein kinase A phosphorylation, Neuron 1996, 17 (4), 759-767.

5. Nehring, R. B., et al. Neuronal inwardly rectifying $\mathrm{K}(+)$ channels differentially couple to PDZ proteins of the PSD-95/SAP90 family, J. Neurosci. 2000, 20 (1), 156-162.

6. Kim, E., and Sheng, M. PDZ domain proteins of synapses, Nat. Rev. Neurosci. 2004, 5 (10), 771-781.

7. Topinka, J. R., and Bredt, D. S. N-terminal palmitoylation of PSD95 regulates association with cell membranes and interaction with K+ channel Kv1.4, Neuron 1998, 20 (1), 125-134.

8. Bravo, J., et al. The crystal structure of the PX domain from p40(phox) bound to phosphatidylinositol 3-phosphate, Mol Cell. 2001, 8 (4), 829-839.

9. Doyle, D. A., et al. Crystal structures of a complexed and peptidefree membrane protein-binding domain: molecular basis of peptide recognition by PDZ, Cell 1996, 85 (7), 1067-1076.

10. Long, J. F., et al. Supramodular structure and synergistic target binding of the N-terminal tandem PDZ domains of PSD-95, $J$. Mol. Biol. 2003, 327 (1), 203-214. 
11. Leonoudakis, D., et al. Protein trafficking and anchoring complexes revealed by proteomic analysis of inward rectifier potassium channel (Kir2.x)-associated proteins, J. Biol. Chem. 2004, 279 (21), 22331-22346.

12. Birrane, G., Chung, J., and Ladias, J. A. Novel mode of ligand recognition by the Erbin PDZ domain, J. Biol. Chem. 2003, 278 (3), 1399-1402.

13. Pegan, S., et al. Cytoplasmic domain structures of Kir2.1 and Kir3.1 show sites for modulating gating and rectification, Nat. Neurosci. 2005, 8 (3), 279-287.

14. Kubo, Y., et al. Primary structure and functional expression of a mouse inward rectifier potassium channel, Nature 1993, 362 (6416), 127-133.

15. Marley, J., Lu, M., and Bracken, C. A method for efficient isotopic labeling of recombinant proteins, J. Biomol. NMR 2001, 20 (1), $71-75$.

16. Clancy, S. M., et al. Pertussis-toxin-sensitive Galpha subunits selectively bind to C-terminal domain of neuronal GIRK channels: evidence for a heterotrimeric G-protein-channel complex, Mol. Cell Neurosci. 2005, 28 (2), 375-389.

17. Pervushin, K., et al. Attenuated T2 relaxation by mutual cancellation of dipole-dipole coupling and chemical shift anisotropy indicates an avenue to NMR structures of very large biological macromolecules in solution, Proc. Natl. Acad. Sci. U.S.A. 1997, 94 (23), 12366-12371.

18. Güntert, P., et al. Processing of multi-dimensional NMR data with the new software PROSA, J. Biomol. NMR. 1995, 2 (6), 619629.

19. Keller, R. (2004) The Computer Aided Resonance Assignment Tutorial, 1st ed., p 81, Cantina Verlag, Goldau.

20. Zuiderweg, E. R., and Fesik, S. W. Heteronuclear threedimensional NMR spectroscopy of the inflammatory protein $\mathrm{C} 5 \mathrm{a}$, Biochemistry 1989, 28 (6), 2387-2391.

21. Griesineger, C., et al. Clean TOCSY for $1 \mathrm{H}$ spin system identification in Macromolecules, J. Am. Chem. Soc. 1988, (110), 78707872 .
22. Grzesiek, S., and Bax, A. Amino acid type determination in the sequential assignment procedure of uniformly $13 \mathrm{C} / 15 \mathrm{~N}$-enriched proteins, J. Biomol NMR. 1993, 3 (2), 185-204.

23. Ritter, C., et al. 3D TROSY-HNCA(coded)CB and TROSYHNCA(coded)CO experiments: triple resonance NMR experiments with two sequential connectivity pathways and high sensitivity, J. Biomol. NMR. 2004, 28 (3), 289-294.

24. Hall, R. A., et al. The beta2-adrenergic receptor interacts with the $\mathrm{Na}+/ \mathrm{H}+$-exchanger regulatory factor to control $\mathrm{Na}+/ \mathrm{H}+$ exchange, Nature 1998, 392 (6676), 626-630.

25. Matsuo, H., et al. Identification by NMR Spectroscopy of Residues at Contact Surfaces in Large, Slowly Exchanging Macromolecular Complexes, Journal of American Chemical Society. 1999, 121 (42), 9903-9904.

26. Tochio, H., et al. Solution structure and backbone dynamics of the second PDZ domain of postsynaptic density-95, J. Mol. Biol. 2000, 295 (2), 225-237.

27. Baker, K. A., et al. NMR-Derived Dynamic Aspects of N-Type Inactivation of a Kv Channel Suggest a Transient Interaction with the T1 Domain, Biochemistry 2006, 45 (6), 1663-1672.

28. Valtschanoff, J. G., and Weinberg, R. J. Laminar organization of the NMDA receptor complex within the postsynaptic density, $J$. Neurosci. 2001, 21 (4), 1211-1217.

29. Gee, S. H., et al. Single-amino acid substitutions alter the specificity and affinity of PDZ domains for their ligands, Biochemistry 2000, 39 (47), 14638-14646.

30. Skelton, N. J., et al. Origins of PDZ domain ligand specificity. Structure determination and mutagenesis of the Erbin PDZ domain, J. Biol. Chem. 2003, 278 (9), 7645-7654.

31. Ma, D., et al. Role of ER export signals in controlling surface potassium channel numbers, Science 2001, 291 (5502), 316-319.

32. Wüthrich, K. (1986) NMR of Proteins and Nucleic Acids, Wiley, Indianapolis.

BI062228Q 